\title{
False recognition and perception without awareness
}

\author{
STEVE JOORDENS and PHILIP M. MERIKLE \\ University of Waterloo, Waterloo, Ontario, Canada
}

\begin{abstract}
Jacoby and Whitehouse (1989) demonstrated that the probability of calling new test words "old" (i.e., false recognition) is biased by context words. When context words were briefly exposed and subjects were not informed of their presence, new words were called "old" more often if the context and test words were identical than if the context and test words were different. When the context words were presented at longer exposure durations and subjects were informed of their presence, the opposite pattern of results occurred. In Experiment 1, we replicated the critical qualitative difference across conditions reported by Jacoby and Whitehouse. In addition, the combined results of Experiments 2 and 3 demonstrated that the exposure duration of the context words, and not the instructions to the subjects, is the primary factor determining which pattern of false recognition occurs. However, in contrast with the findings of Jacoby and Whitehouse, both patterns of false recognition were associated with significant recognition memory for the context words. The latter finding presents problems for any interpretation of false recognition, which implies that the briefly exposed context words are perceived without awareness.
\end{abstract}

Jacoby and Whitehouse (1989) recently reported two experiments that provide compelling evidence for perception without awareness. Basically, their experiments showed that errors in recognition (i.e., false recognition) are differentially influenced by context words that are presented under conditions assumed to reflect aware and unaware states. Given the potential significance of the Jacoby and Whitehouse results for documenting and understanding perception without awareness, the purpose of the present experiments was to further examine the false recognition phenomenon. Before describing either the Jacoby and Whitehouse findings or the present experiments in detail, we will consider earlier approaches to the study of perception without awareness to illustrate why the Jacoby and Whitehouse findings provide such compelling evidence for perception without awareness.

\section{The Dissociation Paradigm}

Almost all evidence supporting the existence of unconscious perception comes from studies based on a version of the dissociation paradigm. The basic logic underlying this paradigm requires comparisons between two different measures of perception. One measure is assumed to reflect consciously perceived information, while the sec-

This research was supported by a Natural Sciences and Engineering Research Council of Canada Scholarship awarded to the first author and by a Natural Sciences and Engineering Research Council of Canada grant (APA-231) awarded to the second author. The authors are indebted to Eyal Reingold for many constructive discussions of the issues and to Jan Williams for writing the software. Correspondence concerning this article should be addressed to either Steve Joordens or Philip M. Merikle, Department of Psychology, University of Waterloo, Waterloo, ON, Canada N2L 3G1. (For e-mail inquiries, use joordens@watserv1. uwaterloo.ca or merikle@watdcs.uwaterloo.ca.) ond measure is assumed to reflect both consciously and unconsciously perceived information. To demonstrate perception without awareness, conditions are found under which the measure of conscious perception indicates null sensitivity. If the second measure of perception can then be shown to reveal greater than null sensitivity, it follows that the second measure reflects information that is not available to consciousness.

A good example of the use of the dissociation paradigm to demonstrate unconscious perception is an early experiment reported by Sidis in 1898 . Sidis assumed that subjects' claims concerning whether or not they could "see" visual stimuli provided the best indicator of awareness. In order to find conditions under which subjects were unaware of stimuli, Sidis systematically increased the distance between subjects and cards containing printed letters or digits until the subjects claimed to no longer "see" what was on the cards. Once the distance at which subjects claimed not to see the stimuli was established, Sidis asked the subjects to guess the identity of the letters and digits. He found that the subjects could guess the identity of the items printed on the cards at a better than chance level of accuracy. Thus, the subjects' guesses indicated that the stimuli were perceived under the same conditions as those under which their reports concerning the visibility of the stimuli indicated that the items on the cards were not perceived. From these findings, Sidis (1898) concluded that information other than that available to the "primary waking self" (p. 171) assisted the subjects' guesses.

Even though the logic underlying the dissociation paradigm is relatively straightforward, the application of the dissociation paradigm to the study of unconscious perception has been problematic. The major problem is a lack of consensus about what constitutes an adequate measure 
of awareness. What some investigators consider to be a measure of perception without conscious awareness, other investigators consider to be a measure of conscious perceptual experience. For example, Sidis (1898) believed that a forced-choice guessing task was sensitive to the information that was not available to conscious awareness. Holender (1986), on the other hand, believes that forcedchoice tasks similar to the one used by Sidis are actually measures of the information available to consciousness. As pointed out by Reingold and Merikle (1988, 1990), much of the controversy surrounding measures of awareness centers on questions concerning whether or not any given measure of conscious awareness provides an exhaustive index of all relevant conscious information. If the selected measure of awareness does not provide an exhaustive index, any sensitivity revealed by a second measure of perception may simply reflect conscious information that the measure of awareness fails to index. Thus, any measure of awareness used within this widely implemented version of the dissociation paradigm must be assumed to provide an exhaustive index of all relevant conscious information.

Unfortunately, it is difficult to justify any measure of perception on an a priori basis as an exhaustive index of conscious experience. This difficulty in justifying measures represents a major problem for studies based on the dissociation paradigm, because the success of such studies is critically dependent on the adequacy of the measure of awareness. Given the lack of knowledge concerning how different measures may index conscious and unconscious perceptual processes, any study that simply demonstrates a dissociation between two measures of perception cannot provide noncontroversial evidence either for or against perception without awareness.

\section{Qualitative Differences}

The difficulty in justifying measures of awareness has led to the use of qualitative differences across aware and unaware conditions as converging evidence for validating particular measures. The logic behind establishing qualitative differences rests on the assumption that perception with awareness should, at least under certain conditions, lead to different consequences than perception without awareness. The importance of this assumption to the study of perception without awareness has been repeatedly emphasized by many investigators (e.g., Cheesman \& Merikle, 1986; Dixon, 1971, 1981; Kelley \& Jacoby, 1990; Marcel, 1983; Merikle \& Reingold, in press; Reingold \& Merikle, 1990; Shevrin \& Dickman, 1980). It follows from this assumption that conditions should exist under which perceiving stimuli with awareness results in a qualitatively different response pattern than does perceiving stimuli without awareness. More importantly, given certain assumptions concerning the nature of consciousness, it should be possible to predict the manner in which behavior will differ following perception with and without awareness. Thus, if perceiving stimuli with awareness has qualitatively different consequences than perceiving stimuli without awareness, such findings can be used to validate a measure of awareness.

An experiment by Marcel (1980) provides a good example of an attempt to use a qualitative difference to validate a measure of awareness. The experiment was based on the idea that a primary function of consciousness is the selection of context-relevant stimulus information. To test this idea, Marcel presented polysemous words with two possible meanings (e.g., PALM) under conditions associated with either null or greater than null sensitivity on a presence/absence discrimination task. In addition, these polysemous words were presented in several contexts. In the congruent context, each polysemous word was preceded and followed by a word related to a single meaning (e.g., HAND, PALM, WRIST), whereas in the incongruent context, the words that preceded and followed each polysemous word were related to two different meanings (e.g., TREE, PALM, WRIST). The subjects' task was to decide whether the third letter string in the sequence of three stimuli was a word or a nonword. The results showed that when the polysemous words were visible, as indicated by better than chance presence/absence discrimination, decisions concerning the third letter string were only facilitated in the congruent context condition. Marcel interpreted this result as indicating that conscious perception of a polysemous word led to the selection of the meaning consistent with the context. However, when the polysemous words were presented under the conditions associated with chance performance on the presence/ absence discrimination task, both the congruent and the incongruent contexts facilitated decisions concerning the third letter string. Marcel interpreted this result as indicating that the context does not constrain the selection of meaning when polysemous words are perceived without awareness. Given these qualitatively different response patterns associated with chance and greater than chance performance, Marcel suggested that the presence/absence discrimination task did in fact provide an adequate measure of awareness in his experiment.

Other investigators have also reported interesting qualitative differences associated with measures assumed to distinguish conscious from unconscious perceptual states (e.g., Cheesman \& Merikle, 1986; Groeger, 1984). Although these demonstrations of qualitative differences are not immune to criticism, they have two distinct advantages over studies based on establishing simple dissociations between measures of perception. The first is that alternative explanations of the results are constrained when a qualitative difference is used as converging evidence for the adequacy of a measure of awareness. The second is that a search for qualitative differences expands the focus of research. By studying situations in which conscious and unconscious processes lead to different outcomes, one can gain new insights into the possible unique contributions of conscious and unconscious processes. These two advantages-an expanded research focus and a reduction in alternative explanations - make approaches based on establishing qualitative differences between 
aware and unaware states superior to any approach based simply on establishing dissociations between two different measures.

\section{False Recognition}

Recently, Jacoby and Whitehouse (1989) reported an interesting qualitative difference between what they consider to be aware and unaware states. Their experiment centered on false recognition that, operationally defined, occurs whenever a subject responds "old" to a new test item on an old/new recognition test. Jacoby and Whitehouse found that different patterns of false recognition $\alpha c$ cur, depending on the context in which a test item appears. More importantly, they also claim that qualitatively different patterns of false recognition occur when subjects are aware or unaware of this context.

The basic method in the Jacoby and Whitehouse (1989) experiments was to use an initial study phase for a large number of words followed by an old/new recognition test. The unique aspect of the recognition test was that each test word was preceded by a briefly presented context word. Across trials, the context word was identical to the test word (match), different from the test word (nonmatch), or a neutral letter string xoxox (baseline). Each subject's awareness of the context words was controlled by varying both the exposure duration of the context words and the instructions. For the aware group of subjects, each context word was exposed for $200 \mathrm{msec}$, and the subjects were told that two words would be presented on each trial. For the unaware group of subjects, on the other hand, each context word was presented for $50 \mathrm{msec}$, and the instructions to the subjects made no reference to the existence of context words. The subjects in the aware group were simply told to categorize each test word.

The results showed that the patterns of false recognition were qualitatively different across the two groups of subjects. The subjects who received the short exposure of the context words and the unaware instructions responded "old" more often to new test words presented on match trials than to new test words presented on nonmatch trials. In contrast, the subjects who received the long exposure of the context words and the aware instructions responded "old" more often to new test words presented on nonmatch trials than to new test words presented on match trials. To establish that these different patterns of false recognition were associated with different levels of awareness for the context words, Jacoby and Whitehouse (1989) measured recognition memory for the context words. The results indicated that the aware subjects recognized some of the context words. However, the unaware subjects could not discriminate context words from new words. Thus, a higher level of false recognition on match than on nonmatch trials was associated with null recognition, and a higher level of false recognition on nonmatch than on match trials was associated with recognition of the context words.

Jacoby and Whitehouse (1989) suggested that these different patterns of false recognition across the aware and unaware conditions reflect different attributions of perceived familiarity. In consistency with the general view of memory and consciousness proposed by Jacoby and his colleagues (e.g., Jacoby \& Kelley, 1987; Kelley \& Jacoby, 1990), Jacoby and Whitehouse assumed that each prior presentation of a stimulus increases its perceptual fluency, which, in turn, increases its perceived familiarity. Given these assumptions, it follows that the test words presented on match trials should have greater perceptual fluency than would the test words presented on nonmatch trials. Thus, the test words presented on match trials should have greater perceived familiarity than would the test words presented on nonmatch trials.

According to Jacoby and Whitehouse (1989), the greater perceived familiarity on match trials than on nonmatch trials was attributed in different ways, depending on whether the subjects were aware or unaware of the context words. Subjects who were aware of the context words attributed the perceived familiarity of the test words to the preceding context words rather than to the initial study phase. Unaware subjects, on the other hand, attributed the perceived familiarity of the test words to the initial study phase rather than to the preceding context words. These different attributions led the unaware subjects to give more "old" responses on match than on nonmatch trials and the aware subjects to give more "old" responses on nonmatch than on match trials. Thus, on the basis of assumptions concerning how aware and unaware subjects attribute perceived familiarity, Jacoby and Whitehouse provided an intriguing account of the qualitatively different patterns of false recognition.

The Jacoby and Whitehouse (1989) study illustrates how qualitative differences can provide compelling evidence for perception without awareness. The different patterns of false recognition across aware and unaware states support two important conclusions. First, the results suggest that recognition memory is an adequate measure of awareness within the context of the dissociation paradigm. Second, both consciously and unconsciously perceived events appear to lead to increased perceptual fluency, but the attributions of perceptual fluency differ, depending on how the event is perceived. Thus, the qualitative difference in false recognition both validates recognition memory as a measure of awareness and provides an indication of the differences that distinguish conscious from unconscious perceptual processes.

\section{Present Experiments}

Given the potential importance of false recognition for the study of perception without awareness, the purpose of the present experiments was to examine the phenomenon in greater detail. The experiments addressed both empirical and conceptual issues. The empirical issues concerned the replicability of the qualitatively different patterns of false recognition and the relative contributions of instructions and exposure duration to these patterns. The conceptual issue centered on the question of whether the different patterns of false recognition were consistently 
associated with null and greater than null recognition memory. A consistent association would constitute compelling evidence for the conceptual distinction between perception with and perception without awareness.

\section{EXPERIMENT 1}

The purpose of Experiment 1 was to replicate the results of Jacoby and Whitehouse's (1989) study. Two groups of subjects were tested; one group received short exposures of the context words and the unaware instructions, and the second group received long exposures of the context words and the aware instructions. The patterns of false recognition across the two groups of subjects were compared to see whether the same qualitative difference as observed by Jacoby and Whitehouse would emerge. Recognition memory for the context words was also tested to see whether different patterns of false recognition were associated with null and greater than null recognition memory. The test of recognition memory was modified from the paper and pencil task used by Jacoby and Whitehouse, so that it was possible to calculate a measure of memory sensitivity.

\section{Method}

Subjects. Thirty-two undergraduate students at the University of Waterlon were recruited from a paid subject pool. All subjects had normal or corrected-to-normal vision, and English was their first language. The subjects were assigned to one of the two groups in an alternating fashion when they arrived at the laboratory for the experiment. Within each group, half of the subjects were female and half of the subjects were male. Each subject was paid $\$ 5$ following completion of the experimental session.

Procedure. The first phase of the experiment was a study phase, in which 96 words were presented to the subjects at a rate of 1 word per second. Each word was presented for $500 \mathrm{msec}$, and the presentation of each word was followed for $500 \mathrm{msec}$ by a blank field. The subjects were instructed to read the words silently to themselves, and they were told that their memory for the words would be tested later in the experiment.

The second phase of the experiment consisted of a test of recognition memory for the words presented during the study phase. Each trial consisted of the following sequence of events: (1) a 500-msec masking stimulus (i.e., \&\&\&\&\&\&\&), (2) a context stimulus, (3) the \&\&\&\&\&\&\& masking stimulus for another $500 \mathrm{msec}$, and (4) a test word. All stimuli were presented at the same screen location, and each test word remained in view until a subject indicated whether it was "old" or "new" with respect to the initial study list.

Three different trial contexts-match, nonmatch, and baselinewere used. On match trials, the context word and the test word were identical. On half of the trials, the word was old (i.e., presented in the study phase), and on the remaining trials, it was new. On nonmatch trials, the context word and the test word were different. The context word was always new, and equal numbers of test words were old and new. On baseline trials, the context word was the letter string xoxox. It was followed equally often by an old or a new test word.

Both the exposure duration of the context words and the instructions were varied across the two groups of subjects. For the unaware group of subjects, the context words were presented for $43 \mathrm{msec}$, and the subjects were not told of the presence of the context words. ${ }^{1}$ Instead, they were told to use the onset of the ampersands as a cue for the subsequent presentation of a test word. In addition, as in the Jacoby and Whitehouse (1989) study, the inter- trial interval was varied randomly (i.e., from 1.8 to $2.8 \mathrm{sec}$ ) to encourage the unaware subjects to use the ampersands as cues. For the aware group of subjects, the context words were presented for $200 \mathrm{msec}$ and the instructions stated explicitly that two words would be presented on each trial. The subjects in the aware group were told to read the first word on each trial silently to themselves and that their memory for these words would be tested later in the experiment. The subjects in both groups were told to categorize each test word as "old" or "new" as quickly and accurately as possible and that their decision times were being recorded.

The test of recognition memory for the study words consisted of a total of 192 trials. The first 12 trials were practice trials, and the old test words for these trials were the first three and last three words of the initial study list. As in the Jacoby and Whitehouse (1989) study, the practice trials for the unaware subjects consisted of baseline trials, and the practice trials for the aware subjects consisted of four exemplars of each of the three trial contexts. Following the completion of the practice trials, all subjects completed 180 test trials, consisting of 90 trials each for old and new test words. Within each group of 90 trials, there were 30 trials of each trial type (i.e., match, nonmatch, and baseline).

The final phase of the experiment consisted of a test of recognition memory for the context words used on the match and nonmatch trials of the test of study-word recognition. A total of 40 context words was selected. Twenty words were selected from the match trials and 20 words were selected from the nonmatch trials. Ten of the words selected from each trial type had been followed by old words. Within these constraints, the selection of words was random. Before beginning this final test of recognition memory, the unaware subjects were told that words had been briefly presented just prior to the presentation of the test words in the immediately preceding test of recognition memory. All subjects were instructed to categorize a word as "old" if it had been presented as a context word on the earlier test.

Materials and Apparatus. A pool of 300 five-letter nouns was compiled from the KuCera and Francis (1967) norms. Word frequencies for these nouns ranged from 8 to 50 occurrences per million. For each subject, 296 words were selected randomly from this pool for use as the stimulus materials.

The words selected for each subject were randomly assigned to the various conditions of the experiment. Ninety-six words were used as study words, and an additional 96 words were assigned as new words on the test of recognition memory for the study words. Another 64 words were used on the nonmatch trials of the recognition test for the study words. Finally, 40 additional words were used as new test words on the test of recognition memory for the context words.

All stimulus materials were presented on a Zenith color monitor (Model ZCM-1490) connected to a Zenith IBM-compatible processor (Model ZDF-2237-BK). The viewing distance was $65 \mathrm{~cm}$. The stimuli were displayed in lowercase gray characters on a black background, and each character was approximately $3 \mathrm{~mm}$ wide by $4 \mathrm{~mm}$ high. All stimuli were centered both horizontally and vertically.

Two touch-sensitive plates were used by the subjects to indicate their decisions on the tests of recognition memory. These plates were located on a table directly in front of the subjects. One plate was labeled OLD, and the other plate was labeled NEW. The subjects were allowed to position these touchplates in a comfortable manner prior to the recognition memory tests.

\section{Results and Discussion}

The primary results in the present experiments were the patterns of false recognition across aware and unaware conditions. However, for the sake of completeness, both the correct recognition and the false recognition data were analyzed. Table 1 shows the mean proportions of false 
Table 1

Mean Proportion False Recognition in Experiments 1, 2, and 3

\begin{tabular}{|c|c|c|c|c|c|c|}
\hline \multirow[b]{3}{*}{ Condition } & \multicolumn{6}{|c|}{ Context } \\
\hline & \multicolumn{2}{|c|}{ Match } & \multicolumn{2}{|c|}{ Nonmatch } & \multicolumn{2}{|c|}{ Baseline } \\
\hline & $M$ & $S E$ & $M$ & $S E$ & $M$ & $S E$ \\
\hline \multicolumn{7}{|c|}{ Experiment 1} \\
\hline Unaware $/ 43 \mathrm{msec}$ & .35 & .02 & .29 & .02 & .31 & .03 \\
\hline Aware $/ 200 \mathrm{msec}$ & .35 & .03 & .43 & .03 & .30 & .03 \\
\hline \multicolumn{7}{|c|}{ Experiment 2} \\
\hline Unaware/57 msec & .42 & .03 & .36 & .02 & .38 & .04 \\
\hline Aware/57 msec & .39 & .03 & .31 & .03 & .33 & .03 \\
\hline \multicolumn{7}{|c|}{ Experiment 3} \\
\hline Aware $/ 57 \mathrm{msec}$ & .37 & .03 & .30 & .02 & .28 & .02 \\
\hline Aware/228 msec & .31 & .03 & .39 & .02 & .27 & .02 \\
\hline
\end{tabular}

Note-For all conditions, match and nonmatch contexts were significantly different at $p<.05$.

Table 2

Mean Proportion Correct Recognition in Experiments 1, 2, and 3

\begin{tabular}{|c|c|c|c|c|c|c|}
\hline \multirow[b]{3}{*}{ Condition } & \multicolumn{6}{|c|}{ Context } \\
\hline & \multicolumn{2}{|c|}{ Match } & \multicolumn{2}{|c|}{ Nonmatch } & \multicolumn{2}{|c|}{ Baseline } \\
\hline & $M$ & $S E$ & $M$ & $S E$ & $M$ & $S E$ \\
\hline \multicolumn{7}{|c|}{ Experiment 1} \\
\hline Unaware/43 $\mathrm{msec} *$ & .59 & .03 & .66 & .03 & .63 & .02 \\
\hline Aware $/ 200 \mathrm{msec}^{*}$ & .58 & .03 & .63 & .03 & .63 & .02 \\
\hline \multicolumn{7}{|c|}{ Experiment 2} \\
\hline Unaware/57 $\mathrm{msec}^{*}$ & .67 & .03 & .60 & .02 & .60 & .02 \\
\hline Aware/57 msec & .68 & .04 & .68 & .03 & .66 & .02 \\
\hline \multicolumn{7}{|c|}{ Experiment 3} \\
\hline Aware/57 msec & .54 & .03 & .60 & .02 & .57 & .02 \\
\hline Aware/228 msec & .54 & .03 & .59 & .02 & .56 & .02 \\
\hline
\end{tabular}

*Match and nonmatch contexts were significantly different at $p<.05$

recognition for all experiments, and the comparable means for correct recognition are shown in Table 2. Even though the effects of context should be similar for both false recognition and correct recognition, one complicating factor with correct recognition is that memory for the prior presentations of the words may either attenuate or interact with the effects of context. Thus, it is not surprising that the effects revealed by the correct recognition data were not as robust or as consistent as the effects revealed by the false recognition data. Jacoby and Whitehouse (1989) also found that the false recognition data were more reliable than the correct recognition data. Given the possible interpretive problems with correct recognition, all data analyses focus on false recognition, and the correct recognition data are not discussed further.

The patterns of false recognition over the three trial contexts on the test of study-word recognition are shown in Figure 1. Examination of these patterns reveals that the subjects who received the unaware instructions and the short exposure of the context words responded "old" to the new words more often on match trials than on nonmatch trials. However, the subjects who received the aware instructions and the long exposures of the context words responded "old" to the new words more often on nonmatch trials than on match trials. These contrasting patterns of false recognition are consistent with those found by Jacoby and Whitehouse (1989) for their unaware and aware subjects, respectively.

The false recognition data were evaluated with a $2 \times 3$ repeated measures analysis of variance. This analysis indicated that the main effect of context $[F(2,60)=3.44$, $p<.039]$ interacted significantly with group $[F(2,60)=$ $8.04, p<.001]$. Further analysis of this interaction revealed that the unaware subjects called new test words "old" more often on match trials than on nonmatch trials $[t(15)=2.62, p<.019]$, and that the aware subjects called new test words "old" more often on nonmatch than on match trials $[t(15)=2.57, p<.021]$.

In order to evaluate recognition memory for the context words, a nonparametric measure of memory strength, $A^{\prime}$, was calculated for each subject. The values of $A^{\prime}$ can range from 0 to 1 , with 0.5 representing chance performance (see Snodgrass \& Corwin, 1988, for a more detailed discussion). When these $A^{\prime}$ 'values were calculated, the hit rates were based on the proportion of "old" responses to the context words presented on the nonmatch trials, and the false alarm rates were based on the proportion of "old" responses to the new words. ${ }^{2}$ The mean hit and false alarm rates associated with the $A^{\prime}$ values are displayed in Table 3.

Analysis of these $A^{\prime}$ values revealed that the mean for the subjects who received the unaware instructions $(M=$ .45 ) did not differ significantly from chance $[t(15)=1.97$, $p>, 05]$, whereas the mean for the subjects who received the aware instructions $(M=.71)$ was considerably better than chance $[t(15)=5.91, p<.001]{ }^{3}$ These results are completely consistent with the findings reported by Jacoby and Whitehouse (1989); their unaware group of subjects displayed chance recognition memory for the context words, whereas their aware group of subjects recog-

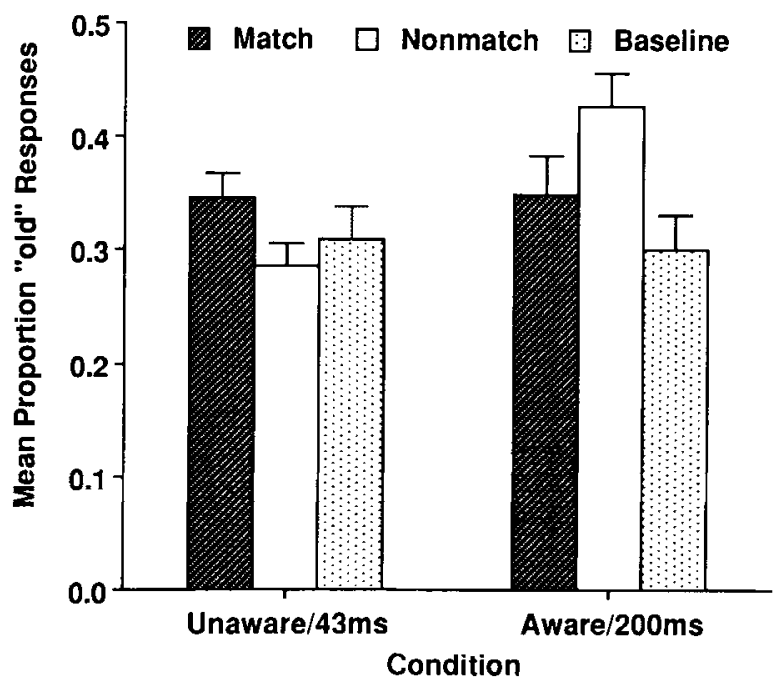

Figure 1. Patterns of false recognition in Experiment 1. (Bars indicate standard errors.) 
Table 3

Mean Hits and False Alarms in Experiments 1, 2, and 3

\begin{tabular}{|c|c|c|c|c|}
\hline \multirow[b]{2}{*}{ Condition } & \multicolumn{2}{|c|}{ Hits } & \multicolumn{2}{|c|}{ False Alarms } \\
\hline & $M$ & $S E$ & $M$ & $S E$ \\
\hline \multicolumn{5}{|c|}{ Experiment 1} \\
\hline Unaware $/ 43 \mathrm{msec}$ & .43 & .04 & .41 & .04 \\
\hline Aware $/ 200 \mathrm{msec}$ & .64 & .04 & .28 & .03 \\
\hline \multicolumn{5}{|c|}{ Experiment 2} \\
\hline Unaware $/ 57 \mathrm{msec}$ & .48 & .05 & .34 & .04 \\
\hline Aware $/ 57 \mathrm{msec}$ & .50 & .04 & .36 & .04 \\
\hline \multicolumn{5}{|c|}{ Experiment 3} \\
\hline Aware $/ 57 \mathrm{msec}$ & .44 & .03 & .33 & .02 \\
\hline Aware $/ 228 \mathrm{msec}$ & .61 & .02 & .33 & .02 \\
\hline
\end{tabular}

nized context words significantly more often than was expected by chance.

The present experiment replicates all essential aspects of the results reported by Jacoby and Whitehouse (1989). The subjects who received the unaware instructions and the short exposure of the context words responded "old" more often when new words were presented in a match than in a nonmatch context. The subjects in this group also displayed chance recognition memory for the context words. On the other hand, the subjects who received the aware instructions and the long exposure of the context words responded "old" more often when new words were presented in a nonmatch than in a match context, and their recognition memory for the context words was considerably better than chance.

Jacoby and Whitehouse (1989) concluded that the chance recognition memory for the context words indicated that these words were perceived without awareness. If one accepts recognition memory as a measure of perceptual awareness, then the criteria necessary for demonstrating perception without awareness within the framework of the dissociation paradigm have been met. Namely, qualitatively different patterns of false recognition are associated with null and greater than null recognition memory. However, there is a confounding factor that may account for the differences in false recognition observed in both the present study and the Jacoby and Whitehouse experiments. The different instructions for the two groups of subjects may have led the subjects in each group to adopt different response strategies. Perhaps all subjects consciously perceived the context words, and the qualitatively different patterns of false recognition, as well as the different levels of recognition memory across the two groups, simply reflect these different response strategies.

Obviously, given these two different interpretations of the different patterns of false recognition, it is important to assess the effects of the aware and unaware instructions independently of exposure duration. To meet this objective, context words were presented for a short exposure duration to two different groups of subjects in Experiment 2; one group received the aware instructions, and the second group received the unaware instructions. If the patterns of false recognition observed in Experi- ment 1 were the result of strategies induced by the instructions, then the aware and unaware subjects in Experiment 2 should exhibit patterns of false recognition similar to those of the aware and unaware subjects in Experiment 1 . However, if the patterns of false recognition observed in Experiment 1 were not the result of the different instructions, then both groups of subjects in Experiment 2 should show a pattern of false recognition similar to the one exhibited by the unaware subjects in Experiment 1.

\section{EXPERIMENT 2}

\section{Method}

Subjects. Thirty-two undergraduate students at the University of Waterloo were recruited from a subject pool. All subjects had normal or corrected-to-normal vision, and English was their first language. Sixteen subjects were assigned to each group in the same manner as in Experiment 1. Within each group, half of the subjects were female. Each subject was paid $\$ 5$ following completion of the experimental session.

Procedure. The method was similar to that of Experiment 1, except for two modifications to the recognition test for study words. First, the exposure duration of the context words was $57 \mathrm{msec}$ for all subjects. The exposure duration was increased from the short, 43-msec exposure duration used in Experiment 1 because the Jacoby and Whitehouse (1989) results suggested that a slightly longer exposure duration may increase the difference between the match and nonmatch trials. The second procedural modification was made with respect to the format of the practice trials. In Experiment 1 , the practice trials for the subjects who received the aware instructions contained four exemplars of each trial context (i.e., match, nonmatch, and baseline), whereas the practice trials for the subjects who received the unaware instructions contained only exemplars of the baseline trial context. In the present experiment, the practice trials for all subjects consisted of four exemplars of each trial context.

\section{Results and Discussion}

The patterns of false recognition over the three trial contexts are shown in Figure 2. Inspection of these patterns reveals that both groups responded "old" to new words more often on match trials than on nonmatch trials. Although the subjects who received the aware instructions showed a slightly larger difference in the proportion of "old" responses on match than on nonmatch trials, the overall pattern of responding was similar across both groups. Thus, the response pattern for both groups of subjects was similar to the response pattern exhibited by the group receiving the unaware instructions in Experiment 1.

The data were evaluated by a $2 \times 3$ repeated measures analysis of variance. The analysis revealed a significant main effect of context $[F(2,60)=4.60, p<.014]$. Individual comparisons revealed that the proportion of "old" responses to new test words was significantly higher on match than on nonmatch trials both for the subjects who received the aware instructions $[t(15)=2.32$, $p<.035]$ and for the subjects who received the unaware instructions $[t(15)=2.42, p<.028]$. Neither the main effect of instructions $[F(1,30)=1.32, p>.25]$ nor the interaction between instructions and context $[F(2,60)=$ $.16, p>.85$ ] was significant. 


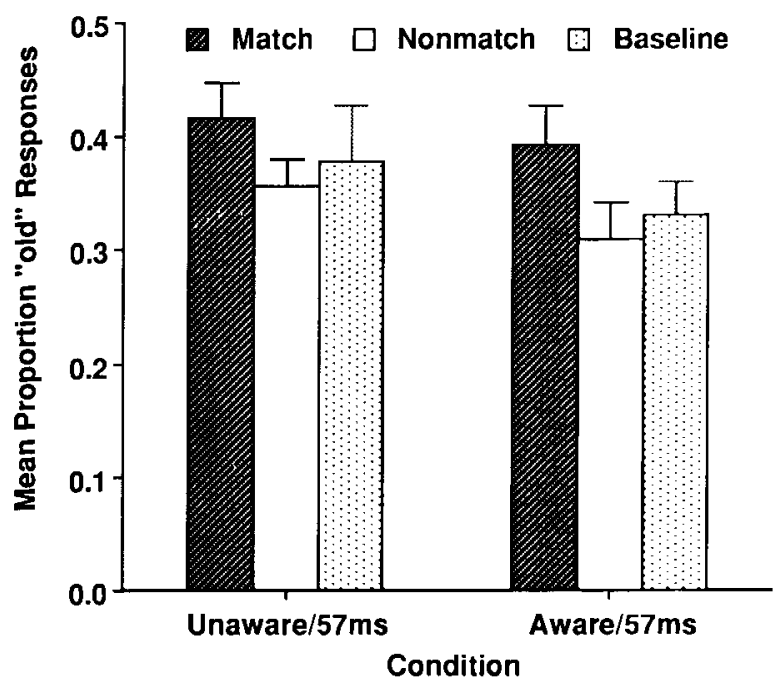

Figure 2. Patterns of false recognition in Experiment 2. (Bars indicate standard errors.)

In contrast with the results of Experiment 1, recognition of the context words was above chance for both the subjects who received the aware instructions ( $M=.62$ ) and the subjects who received the unaware instructions $(M=.61)$. As suggested by the means, an independent $t$ test indicated that recognition did not differ across the two groups $[t(30)=.19, p>.85]$. However, for both groups of subjects, the context words were recognized significantly better than would be expected on the basis of chance [both $t \mathrm{~s}(15)>3.14, p<.01$ ]

The results of this experiment show that when context words are exposed for a short duration, false recognition is greater on match than on nonmatch trials, independently of whether the subjects are given the aware or the unaware instructions. Thus, the pattern of results for both groups of subjects was similar to the one found by Jacoby and Whitehouse (1989), as well as to the one found in Experiment 1 when context words were exposed for the short duration. Given these findings, the higher false recognition on match than on nonmatch trials following briefly presented context words is not due to response strategies associated with the different instructions.

However, the significant recognition memory for the context words raises serious questions concerning the implications of false recognition for perception without awareness. According to the logic underlying the use of the qualitative differences to demonstrate perception without awareness, performance on the measure used to in dex awareness-recognition memory-should predict the pattern of false recognition. More specifically, the pattern associated with perception without awareness should occur only when recognition memory indicates chance performance. In the present experiment, the pattern of false recognition assumed to reflect perception without awareness occurred when recognition memory was significantly better than chance. Thus, the implications of false recognition for perception without awareness are considerably less straightforward than was originally suggested by Jacoby and Whitehouse (1989), because the pattern of false recognition assumed to reflect perception without awareness is not limited to conditions that lead to null recognition.

A comparison of the results of Experiments 1 and 2 suggests that the primary variable determining which pattern of false recognition is observed is the exposure duration of the context words. The conditions for testing the groups receiving the aware instructions in Experiments 1 and 2 were identical in all respects except for the exposure duration of the context words. However, the results indicate qualitatively different patterns of false recognition; with the short exposure of the context words in Experiment 2, false recognition was higher on match than on nonmatch trials, whereas with the long exposure of the context words in Experiment 1, false recognition was higher on nonmatch than on match trials. Thus, the qualitative difference in patterns of false recognition seems to depend solely on the exposure duration of the context words.

Given the analysis above, the primary goal of Experiment 3 was to evaluate directly the effect of exposure duration of the context words on the pattern of false recognition. To meet this objective, the short and long exposures of the context words were presented on a random basis to a single group of subjects. This procedure eliminated any possibility of the results' being contaminated by differences in strategy across the two exposure durations. As in the preceding experiments, the relation between the observed patterns of false recognition and recognition memory for the context words was also evaluated.

\section{EXPERIMENT 3}

\section{Method}

Subjects. Thirty-two undergraduate students at the University of Waterloo were recruited from one of two subject pools. Eight subjects received course credit for participation, and the remaining 24 subjects were paid $\$ 5$ on completion of the experiment. All subjects had normal or corrected-to-normal vision, and English was their first language. Half of the subjects were female.

Procedure. In general, the method was similar to the one followed in Experiment 2 for the subjects who received the aware instructions. However, in order to use a within-subject design and to maintain measurement precision, several alterations were made. In the study phase, 126 words were presented to each subject, rather than the 96 words used in Experiment 2. The number of trials on the recognition test of study words was also increased from 180 to 240 trials. On 120 of these 240 trials, the context word was presented for $57 \mathrm{msec}$; on the remaining 120 trials, the context word was presented for $229 \mathrm{msec}$. The exposure duration of the context word on any specific trial was randomly determined, within the constraint that no more than three consecutive trials had the same exposure duration. Within each exposure duration, the 120 recognition trials were further divided into 60 trials with old test words and 60 trials with new test words, and within each group of 60 trials, there were 20 trials of each trial type (match, nonmatch, and baseline).

The number of recognition trials for the context words was also increased. In the present experiment, 160 trials were used rather than the 80 trials used in Experiment 2. These 160 trials were com- 
posed of 80 old and 80 new trials. The words presented on the 80 old trials were the context words that appeared on the nonmatch trials at each exposure duration.

To accommodate the increased number of trials, a larger stimulus pool was used. This pool consisted of 540 words, and the frequency of the words ranged from 3 to 60 occurrences per million (Kucera \& Francis, 1967). For each subject, 416 words were randomly selected from this pool and assigned to conditions following a method similar to the one used in Experiment 1.

\section{Results and Discussion}

The patterns of false recognition over the three trial contexts are shown in Figure 3. At the short exposure of the context words, subjects responded "old" to new words more often on match than on nonmatch trials. However, at the long exposure of the context words, subjects responded "old" to new words more on nonmatch than on match trials. Thus, qualitatively different patterns of false recognition were associated with the short and the long exposures of the context words.

The data were analyzed with a $2 \times 3$ repeated measures analysis of variance. The analysis revealed a significant main effect of context $[F(2,62)=5.92, p<.004]$. The context $\times$ exposure duration interaction was also significant $[F(2,62)=9.81, p<.001]$. Closer examination of this interaction confirmed that false recognition was higher on match than on nonmatch trials when the context words were exposed for the short duration $[t(31)=2.30, p<$ .028 ] and that false recognition was higher on nonmatch than on match trials when the context words were exposed for the long duration $[t(31)=2.80, p<.009]$. Thus, these results confirm that the exposure duration of the context words is the critical variable determining the observed pattern of false recognition.

Recognition memory for the context words was also evaluated across the two context-word exposure durations. As expected, recognition memory was higher when the context words were presented for the long duration $(M=$

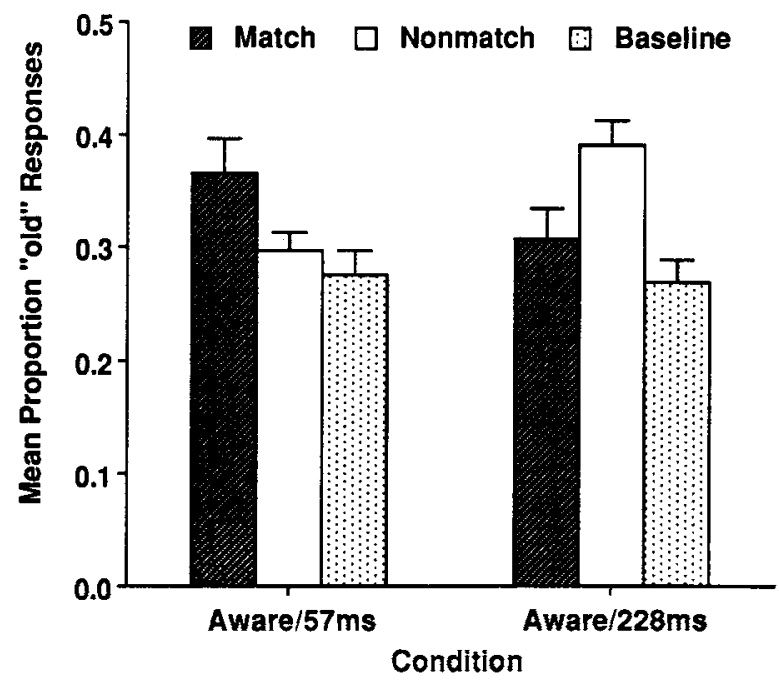

Figure 3. Patterns of false recognition in Experiment 3. (Bars indicate standard errors.)
$.72)$ than for the short duration $(M=.60)[t(31)=8.19$, $p<.001$ ]. More importantly, independent $t$ tests also revealed that both levels of recognition memory were significantly greater than chance [both $t \mathrm{~s}(31)>4.5, p<$ .0011 . Thus, as implied by the results of Experiment 2, the different patterns of false recognition are not associated with null and greater than null recognition performance.

\section{GENERAL DISCUSSION}

The present experiments demonstrate that the different patterns of false recognition reported by Jacoby and Whitehouse (1989) occur reliably. In addition, whereas Jacoby and Whitehouse varied both instructions and the exposure duration of the context words to produce the different patterns of false recognition, the present results show that a variation in exposure duration alone is sufficient to produce the different patterns. Thus, the falserecognition phenomenon is robust, and it can be demonstrated with procedures that are both simpler and more straightforward than the procedures used by Jacoby and Whitehouse.

Even though the different patterns of false recognition can be demonstrated easily, the theoretical implications of false recognition are not clear. Jacoby and Whitehouse (1989) thought that the different patterns of false recognition reflected perception with and perception without awareness. As converging evidence to justify this conclusion, they emphasized that the different patterns of false recognition were associated with null and greater than null recognition for the context words. However, in the present experiments, the different patterns of false recognition were not necessarily associated with null and greater than null recognition. In fact, both patterns of false recognition occurred reliably under stimulus presentation conditions that allowed recognition of the context words (cf. Bernstein \& Welch, 1991). Thus, the converging evidence used by Jacoby and Whitehouse to justify their conclusion is not valid. Without the converging evidence provided by an adequate measure of awareness, the implications of the qualitatively different patterns of false recognition for perception without awareness are considerably less compelling than was suggested by Jacoby and Whitehouse.

Despite the fact that the implications of false recognition for perception without awareness are not as straightforward as was originally assumed, the possibility that the different patterns of false recognition reflect awareness and nonawareness of the context words has intuitive appeal. Subjectively, it is very difficult to "see" the context words exposed for the short duration, whereas context words exposed for the long duration are clearly visible. In previous studies, subjective measures have been used successfully to distinguish between aware and unaware states (e.g., Cheesman \& Merikle, 1986; Merikle \& Reingold, 1990). Perhaps the different patterns of false recognition are associated with null and greater than null sensitivity on a subjective measure. If evidence can be found to indicate that the different patterns of false rec- 
ognition are predicted by a subjective measure of awareness, the intuitive appeal of false recognition as a demonstration of perception without awareness would be realized in experimental evidence.

\section{REFERENCES}

BernStein, I. H., WELCH, K. R. (1991). Awareness, false recognition, and the Jacoby-Whitehouse effect. Journal of Experimental Psychology: General, 120, 324-328.

Chemsman, J., Merukie, P. M. (1986). Distinguishing conscious from unconscious processes. Canadian Joumal of Psychology, 40, 343-367.

DixoN, N. F. (1971). Subliminal perception: The nature of a controversy New York: McGraw-Hill.

Dixon, N. F. (1981). Preconscious processing. Chichester, U.K.: Wiley.

Groeger, J. A. (1984). Evidence of unconscious semantic processing from a forced error situation. British Joumal of Psychology, 75, 305-314.

Holender, D. (1986). Semantic activation without conscious identification in dichotic listening, parafoveal vision, and visual masking A survey and appraisal. Behavioral \& Brain Sciences, 9, 1-23.

JACOBY, L. L., KELLEY, C. M. (1987). Unconscious influences of memory for a prior event. Personality \& Social Psychology Bulletin, $13,314-336$

JACOBY, L. L., Whitehouse, K. (1989). An illusion of memory: False recognition influenced by unconscious perception. Journal of Experimental Psychology: General, 118, 126-135.

KeLLeY, C. M., JACOBY, L. L. (1990). The construction of subjective experience: Memory attributions. Mind \& Language, 5, 49-68.

KuČera, H., a Francis, W. N. (1967). Computational analysis of present day American English. Providence, RI: Brown University Press.

MARCRL, A. J. (1980). Conscious and preconscious recognition of polysemous words: Lacating the selective effects of prior verbal context. In R. S. Nickerson (Ed.), Attention and Performance VIII (pp. 435 457). Hillsdale, NJ: Erlbaum.
MARCEL, A. J. (1983). Conscious and unconscious perception: Experiments on visual masking and word recognition. Cognitive Psychology, 15, 197-237.

Merikle, P. M., Reingold, E. M. (1990). Recognition and lexical decision without detection: Unconscious perception? Joumal of Experimental Psychology: Human Perception \& Performance, 16, 574-583.

MerikLe, P. M. Reingold, E. M. (in press). Measuring unconscious perceptual processes. In R. F. Bornstein \& T. S. Pitman (Eds.), Perception without awareness: Cognitive, clinical, and social perspectives. New York: Guilford.

Reingold, E. M., MeruKLe, P. M. (1988). Using direct and indirect measures to study perception without awareness. Perception \& Psychophysics, 44, 563-575.

Reingold, E. M., Merixte, P. M. (1990). On the inter-relatedness of theory and measurement in the study of unconscious processes. Mind \& Language, 5, 9-28.

Shevrin, H., \& Dickman, S. (1980). The psychological unconscious: A necessary assumption for all psychological theory? American Psychologist, 35, 421-434.

SidIs, B. (1898). The psychology of suggestion. New York: Appleton. SNOdGRass, J. G., \& CoRWIN, J. (1988). Pragmatics of measuring recognition memory: Applications to dementia and amnesia. Journal of Experimental Psychology: General, 117, 34-50.

\section{NOTES}

1. The $43 \mathrm{msec}$ reflect three raster sweeps on a $70-\mathrm{Hz}$ monitor.

2. Context words presented on match trials were not included, because these words, by definition, were also test words.

3. Given that $A^{\prime}$ is nonlinear, mean $A^{\prime}$ values differ slightly from $A^{\prime}$ values calculated directly from the mean hits and false alarms in Table 3 .

(Manuscript received May 24, 1991; revision accepted for publication August 26, 1991.) 